\title{
COMMENTARY
}

\section{Beyond the avoidance of waste: the ethical imperative to focus on value in the NICU}

\author{
BH Arzuaga ${ }^{1,2}$ \\ Journal of Perinatology (2017) 37, 622-623; doi:10.1038/jp.2017.34
}

In the neonatal intensive care unit (NICU), like in all intensive care units (ICUs), concern over the cost of a needed intervention should never come into consideration to the individual physician. In reality, if the question of potential waste is suggested in an ICU, the therapy or intervention under consideration is not likely to be in the best interests of the patient. In life and death, life-saving measures should never be deemed too expensive or too resource intensive.

The Choosing Wisely campaign, which launched in 2012, tasked specialty societies with the creation of five-item - 'Top Five' - lists of therapies or interventions which could be easily identified as 'wasteful' in an effort to eliminate the proverbial low-hanging fruit of medical overuse. The campaign focuses almost exclusively on 'not doing' unnecessary tests or procedures, thereby avoiding waste, cutting costs, and theoretically providing more efficient patient care. ${ }^{1}$ To date, over 70 societies have produced such lists (http://www.choosingwisely.org/clinician-lists/) and in 2015, the American Academy of Pediatrics' Section on Neonatal-Perinatal Medicine (SONPM) added its contribution. ${ }^{2}$ This list, like its predecessors, identifies five commonly utilized diagnostics and therapies in the NICU with little or no appreciable benefit to patient outcomes and suggests that these not be routinely used. This initial step at reducing ineffective medical practices serves as a solid foundation for further discourse on waste reduction in the NICU, but it also raises some important ethical considerations. Specifically, it lends the questions of how physicians can simultaneously balance the utilitarian model of medicine which the Choosing Wisely framework instills with the contemporary emphasis on parental autonomy and shared decision-making and, consequently, how does the individual doctor-patient/doctorfamily relationship fit within the framework?

The type of waste avoidance advocated by Choosing Wisely is fundamentally utilitarian: by reducing unnecessary (including marginally unnecessary) treatments and procedures on individual patients, the patient population as a whole will benefit-the greatest good for the greatest number. A prime example of this, taken from the SONPM list, is the elimination of the practice of obtaining routine term-equivalent or pre-discharge brain magnetic resonance imaging (MRI) studies on premature infants. Routine MRIs, it is argued, are expensive and, while they correlate with neurodevelopmental outcomes of former premature infants at 2 and 5 years of age, they do nothing to improve those outcomes. On the surface, this seems appropriate. If we cannot improve the neurodevelopmental outcome for a particular patient by doing a test, then why do it at all? However, this conclusion ignores another notable measurable outcome that obtaining a pre-discharge MRI may affect: parental anxiety.
Consider what a provider should do when a patient's parents, who may have read about the use of MRls on the internet, strongly request one as hospital discharge approaches? Should the physician accede to their request? Would the decision change if the infant has a history of normal head ultrasounds versus abnormal scans? Should head ultrasound results, which are usually, at best, a crude method of risk-assessment, even matter in the face of parental request rooted in worry over their child's future?

Our duty to our individual patients and families can only be fully upheld within the idea of overuse reduction if the primary focus is shifted from Choosing Wisely's emphasis on pure waste avoidance toward one on value-optimization in the medical care provided to critically-ill newborns. If value can be defined as achieving the best outcome as efficiently as possible, ${ }^{3}$ then examining the impact of specific practices on monetary costs alone not only falls short of helping to define what our actual patient-centered value-based goals should be, but will also become more difficult as more units move towards a system of bundled payments. Instead, in order to optimize value, one must take a multifaceted approach which includes (1) avoiding procedures and treatments with no value; (2) avoiding procedures and treatments with little or marginal value; (3) shifting focus to procedures and treatments with the most benefit, and most importantly; (4) structuring the system in such a way that the money and resources saved by the first and second components are allocated to the patient population from which they were taken.

The Choosing Wisely framework tackles the first and second facets of value-optimization with its 'Top Five' lists but does not address the last two, which may well lead to the campaign being ignored by providers who feel a primary duty to their individual patients in day-to-day practice. Alternatively, a medical association supported shift from a culture of waste avoidance to an environment focused on interventions that provide a patient with the most benefit would invariably succeed in attaining individual providers' acceptance as it falls directly in-line with physicians' fundamental fiduciary roles to their patients. Such a shift is possible by paying attention to what should be done for a patient, rather than what should not. Focusing energies on ways to strengthen the partnership found in successful doctor-family relationships will, in turn, promote shared decision-making, family engagement in medical care, and maybe even increased usage of underutilized resources like neonatal palliative care. For example, actively engaging families in the understanding and consequential management of gastroesophageal reflux will naturally lead to parental adherence to long-term plans. After all, what good is avoiding an anti-reflux medication in the NICU (item \#1 on SONPM's 'Top Five' list) if the family pediatrician will prescribe it on the first post-discharge follow-up visit? 
It is no secret that all intensive care is expensive, and neonatal intensive care is no exception. However, unlike care in adult ICUs where the most money is spent on dying patients, the vast majority of expenditure in the NICU comes from infants who eventually survive. ${ }^{4}$ The average former 25 -week gestational age infant will have an initial hospital stay of approximately 105 days and so for this population in particular there is an ethical imperative to increase the value of the care they receive during that time. By doing so, providers will be motivated to decrease unnecessary interventions that have the potential to cause harm (that is, the use of anti-reflux medications or extended courses of antibiotics in premature infants) and to seek those interventions that will provide the best outcomes for patients, ${ }^{5,6}$ all while simultaneously fostering strong doctor-family relationships, parental autonomy, and shared decision-making.

As wasteful practices are stripped away and the focus begins to shift to more valuable care, it is imperative that overarching systems are able to ensure that money and resources saved by more valuable NICU care are funneled into services that will benefit NICU infants and their families. If not, the scales could tip further away from a model which supports distributive justice and this population could become exploited and marginalized. ${ }^{7}$ It has been shown that premature infants are not perceived to hold the same moral status in our society as other types of patients ${ }^{8}$ and so, if we are not careful, it may become easy for resources no longer being used in the NICU to go on to be allocated to other patient populations.

\section{CONFLICT OF INTEREST}

The author declares no conflict of interest.

\section{REFERENCES}

1 Cassel CK, Guest JA. Choosing Wisely: helping physicians and patients make smart decisions about their care. JAMA 2012; 307(17): 1801-1802.

2 Ho T, Dukhovny D, Zupancic J, Goldmann DA, Horbar JD, Pursley DM. Choosing Wisely in newborn medicine: five opportunities to increase value. Pediatrics 2015; 136(2): e482-e489.

3 Lee TH. Putting the value framework to work. N Engl J Med 2010; 363(26): 2481-2483.

4 Meadow W, Lantos J. Moral reflections in neonatal intensive care. Pediatrics 2009; 123(2): 595-597.

5 Tilburt JC, Cassel CK. Why the ethics of parsimonious medicine is not the ethics of rationing. JAMA 2013; 309(8): 773-774.

6 Brody $\mathrm{H}$. From an ethics of rationing to an ethics of waste avoidance. $N$ Engl J Med 2012; 366(21): 1949-1951.

7 Porter ME. A strategy for health care reform - toward a value-based system. $N$ Engl $J$ Med 2009; 361(2): 109-112.

8 Janvier A, Leblanc I, Barrington KJ. The best-interest standard is not applied for neonatal resuscitation decisions. Pediatrics 2008; 121(5): 963-969. 